\title{
Simulation Based Analysis of Scramble Crossings at Signalized Intersections
}

\author{
Tran $\mathrm{Vu} \mathrm{Tu}^{1}$ and Kazushi Sano ${ }^{2}$ \\ ${ }^{1,2}$ Department of Civil and Environmental Engineering, Nagaoka University of \\ Technology, Niigata, 940-2188, Japan \\ trvutu@stn.nagaokaut.ac.jp
}

\begin{abstract}
This paper conducts an analysis of the effect of scramble crossings in comparison with that of normal ones on the intersection performance by using simulation models. Based on real data collected at a signalized intersection with normal crossings, the research firstly designs and simulates the scenario of scramble crossings at the studied intersection. The paper secondly analyses the effects of the scramble crossings and that of the normal ones under various situations of traffic flow and pedestrian demand. The paper results show that the scramble crossings keep the intersection level of service more stable than the normal ones once the situations of traffic and pedestrian change. In terms of vehicle delay reduction, the pedestrian demand plays an important role in the decisions of choosing the crossing type. The higher the pedestrian demand is, the more advantageous the scramble crossings are. The scramble crossings can reduce the vehicle delay significantly, by up to $35.74 \%$ compared with the normal pedestrian crossings.
\end{abstract}

Keywords: Simulation, Scramble crossing, Diagonal crossing, Pedestrian

\section{Introduction}

\subsection{Research Studies Related to Pedestrian}

Pedestrians play a more and more important role in modern societies where public transportation operates as a key role to ease traffic congestion. The attractiveness of the public transportation encourages walking in any traffic system. At signalized intersections, the interactions between crossing pedestrians and turning traffic streams affect the performance of the intersections. Intuitively, these interactions become significant when the number of crossing pedestrians is high. To improve the situation, scramble crossings or diagonal crossings are promising deployments which have been applied in many countries such as America, Canada, Japan, etc. However, research studies on scramble crossings are still few. Most of the previous studies utilized real applications of scramble crossings to conclude the bi-directional effects between pedestrians and vehicles or to find related pedestrian behaviour. Some others developed simulation models to investigate the effects of scramble crossings. However, the scopes of these research studies have limitations. A criterion for city planners to decide the deployment of scramble crossings at a signalized intersection is still a question mark.

Although there have been many research studies about the effects of pedestrians, pedestrian behaviour, pedestrian safety, etc., in urban streets [26]. Very few research studies about integration of pedestrians into simulation models to investigate the scramble crossings are available. Indeed, most of the previous studies about pedestrians concentrated on pedestrian behavior or pedestrian safety $[5,8,9,29,30,32,33]$. Some research studies focus 
on capacity analysis under the effects of pedestrian flow [3, 27]. These analyses were conducted for Capacity of Right-Turn Movements at Signalized Intersections [28], or for capacity of entire the signalized intersection with the presence of pedestrians or bicycles [4], $[14,28]$. Some others analyzed the conflicts between Right-Turning Vehicles and Pedestrians at urban Signalized Intersections [24], or took pedestrian flow analyses [20], or studied on Pedestrian Impedance of Turning-Movement Saturation Flow Rates [16]. Several papers concluded the benefit based on real applications [7] or developed simulation models for the interaction of vehicles and pedestrians to investigate trade-offs between vehicular and pedestrian traffic $[2,15,25,31]$. However, these studies stopped at investigating into impacts on pedestrians as well as how the intersections perform under the confliction between pedestrians and vehicles. Investigations or analyses concerning scramble crossings that are very useful for the current tendency of developing a pedestrian-friendly environment were not mentioned in these above research studies. Therefore, studies on the benefit of scramble crossings are necessary and urgent.

Concerning scramble crossings, some research studies summarized the consequence of conducting real study cases of scramble deployment [1, 7, 23]. However, these research studies only ends at evaluation of the scramble crossing pedestrian in terms of determinations of the number of pedestrian violations, the safe areas as well as conclusions on the rate of pedestrian-vehicle conflicts, traffic delay or pedestrian delay, etc. Also relating to scramble crossings, Muhammad [15] conducted micro-simulated comparisons of alternative signalized pedestrian crossings including diagonal crossings by using VISSIM. However, the research stops at studying the effects of four different signalized pedestrian crossing technologies on various measures of effectiveness. A single set of vehicle and pedestrian demand could not represent the dynamic traffic and pedestrian situations. In addition, the criteria of choosing pedestrian crossing type have not been mentioned much in previous research studies. Therefore, a study on the effects of scramble crossings in comparison with the normal pedestrian crossings as well as the criteria for determination of the scramble crossings is necessary for the development of a pedestrian-friendly environment.

\subsection{Research Objective}

The objective of this paper aims at two targets. The first target is the simulation of scramble crossings in comparison with the current normal pedestrian crossings at a signalized intersection in Nagaoka city of Japan. The second target is the analysis of the operation of scramble crossings under various situations of traffic flow and pedestrian demand. Based on the analysis, the research then suggests criteria for choosing scramble crossings in terms of vehicle delay reduction.

\section{Methodology}

\subsection{Evaluation Method}

The vehicle delay at the studied intersection is output based on simulation models in PARAMICS. To investigate the signalized intersection performance, the research adopts the Level of Service (LOS) for intersections introduced in HCM 2010 [26]. The table is expressed as follows: 
Table 1. Level of Service

\begin{tabular}{|c|c|}
\hline Level of service & Vehicle delay (s/veh) \\
\hline A & $\mathrm{d} \leq 10$ \\
\hline B & $10<\mathrm{d} \leq 20$ \\
\hline C & $20<\mathrm{d} \leq 35$ \\
\hline D & $35<\mathrm{d} \leq 55$ \\
\hline E & $55<\mathrm{d} \leq 80$ \\
\hline F & $\mathrm{d}>80$ \\
\hline
\end{tabular}

\subsection{Integration of Pedestrians into Simulation Models in PARAMICS}

Quadstone PARAMICS [18] allows simulating traffic and pedestrians for operational assessment of current and future scenarios of traffic conditions including car, bus, tram, cyclist, pedestrian, etc. By using the PARAMICS Pedestrian Module (PPM), the research simulates the actions as well as interactions of autonomous individuals on the traffic system. According to Quadstone PARAMICS [18], signalized pedestrian crossings can be modeled using the concepts of agent type, agent space, region, connector, etc. The interaction between pedestrians and vehicles is analysed through the concepts of shared spaces and vehicle aware space. First of all, the research defines a study area of pedestrians at the studied intersection. The Space types are then defined in the study area, including pedestrian space, shared aggressive space, vehicle aware space, etc. The demand regions are defined to input the demand of pedestrians at each approach of each intersection. These demand regions may specify a source point for agents to be released onto a network or a destination point for agents to be removed from the network. Snapshots at the study site in PARAMICS for the scenario of normal crossings and that of scramble crossings are as shown in Figure 1 and Figure 2.

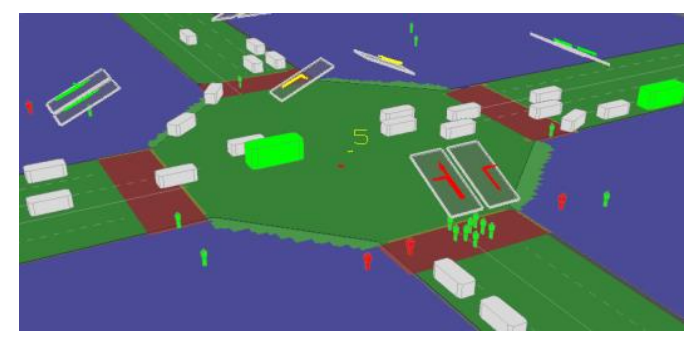

Figure 1. Normal Crossings in PARAMICS

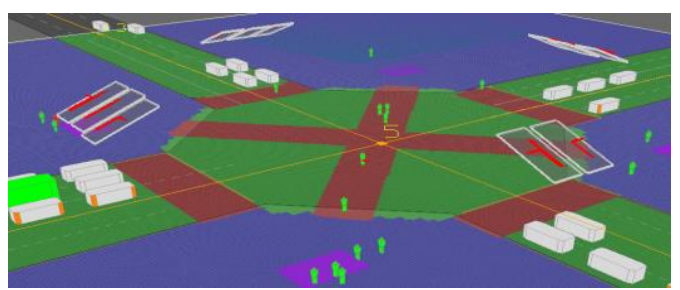

Figure 2. Scramble Crossings in PARAMICS

\subsection{Signal Phases for Pedestrian Crossings}

The current intersection has a cycle of $130 \mathrm{~s}$, consisting of 4 phases as illustrated in Table 2. 
Table 2. Current Phases for Normal Crossings

\begin{tabular}{|c|c|c|c|}
\hline \multicolumn{4}{|c|}{ Current normal pedestrian crossings } \\
\hline \multicolumn{2}{|c|}{ West-East phase $(1,2)$} & \multicolumn{2}{|c|}{ North-South Phase $(3,4)$} \\
\hline 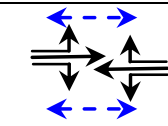 & $\underset{\leftarrow \rightarrow}{\stackrel{\leftrightarrow}{*}}$ & i & 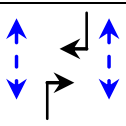 \\
\hline $48(\mathrm{~s})$ & $13(\mathrm{~s})$ & $55(\mathrm{~s})$ & $14(\mathrm{~s})$ \\
\hline Traffic flow & $\longrightarrow$ & Pedestrian & $\leftrightarrow->$ \\
\hline
\end{tabular}

Concerning the ways of estimating signal time for scramble crossings, there have been many approaches $[12,33]$. However, each approach has its shortcomings. For example, $\mathrm{Z}$. Yang et al., [33] proposed an optimization model to determine pedestrian crossing phases based on minimizing vehicle and pedestrian delays by genetic algorithm. However, the most important thing concerning pedestrians is safety was not mentioned. For the sake of simplicity, this research uses the minimum green interval to ensure the walking time introduced in Highway Capacity Manual [26]. The minimum walking time depends on walking speed, effective crosswalk, number pedestrian and the road width ( $\mathrm{w}>3 \mathrm{~m})$ as shown in the following equation.

$$
G_{\min , i}=3.2+\frac{L_{i}}{v_{p}}+\left(2.7 \frac{n_{p}}{w_{e}}\right)
$$

where,

Li: the width of the intersection at approach i (m)

vp: the average walking speed $(\mathrm{m} / \mathrm{s})$

we: the effective width of the crosswalk (m)

np: the number of pedestrians

Depending on the geometrical feature at the study site, the scramble crossings are proposed to be $36.5 \mathrm{~m}$ long at the studied intersection. The information of pedestrian such as the maximum number of pedestrians in a group, walking speed, etc. is collected from the real observation. In this research, the average walking speed is $1.64 \mathrm{~m} / \mathrm{s}$; the start up lost time is assumed to be $2 \mathrm{~s}$. The proposed phases for scramble pedestrians are shown in Table 3.

Table 3. Proposed Phases for Scramble Crossings

\begin{tabular}{|c|c|c|c|c|}
\hline \multicolumn{5}{|c|}{ Proposed scramble pedestrian crossings } \\
\hline \multicolumn{2}{|c|}{ West-East phase } & \multicolumn{2}{|c|}{ North-South Phase } & \multirow{2}{*}{ 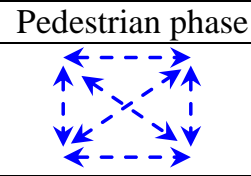 } \\
\hline 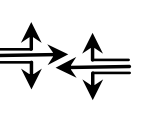 & $\checkmark$ & & $\vec{P}$ & \\
\hline $35(\mathrm{~s})$ & $13(\mathrm{~s})$ & $41(\mathrm{~s})$ & $14(\mathrm{~s})$ & $27(\mathrm{~s})$ \\
\hline Traffic flo & \multicolumn{2}{|c|}{$\longrightarrow$} & \multicolumn{2}{|c|}{ Pedestrian flow $\longleftrightarrow-\rightarrow$} \\
\hline
\end{tabular}




\section{Study Location}

The studied intersection is Otetsuri intersection, lying on Route No. 36 leading to Nagaoka Station. This intersection is near Nagaoka station where there are lots of pedestrians moving. According to a survey [22], Nagaoka city has a large network of bus routes with Nagaoka Station as a hub. The station serves approximately 1500 buses operating, 11,600 railway passengers and 25,000 bus users everyday. In addition, from April 1st 2012, Nagaoka city Hall Plaza was setup near the intersection. This City Hall Plaza consists of the City Office, Arena, the Roofed Open Plaza (Nakadoma), and many other facilities providing various spaces for citizens to come together. Therefore, a huge number of pedestrians is predicted to walk around here. Moreover, there are many restaurants, shopping centres, bus stops around the intersection and along the road No. 351. The studied intersection as well as the four entry approaches is as illustrated in Figure 3.

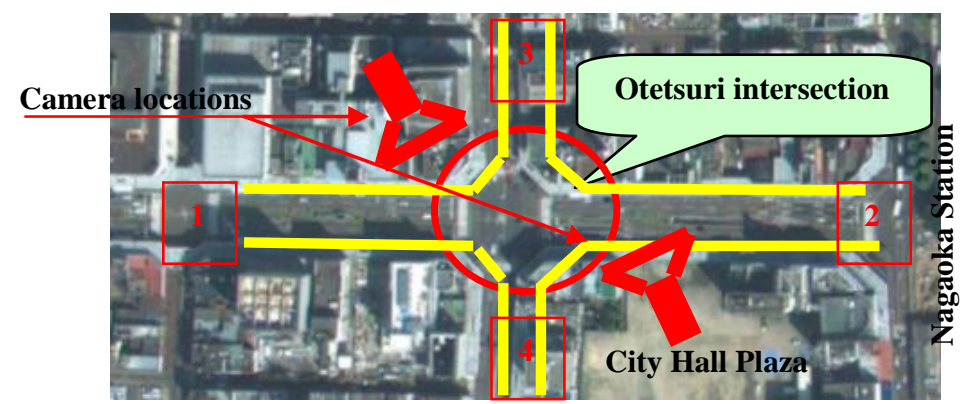

Figure 3. The Studied Intersection

The research used cameras to record the traffic and pedestrian flow as shown in Figure 3 during the peak hour from 8AM-9AM. The recorded videos are analyzed in Transportation Lab by using a video based software (SEV) [13]. Based on the result of data analysis, the traffic proportion at the studied site includes bus, car, small truck and big truck. Besides bus routes, the traffic proportion with the figures is shown as in Figure 4.

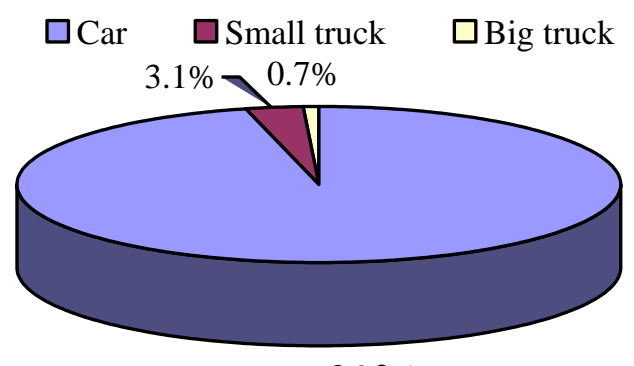

$96.2 \%$

Figure 4. Traffic Proportion at the Studied Site

Because crossing pedestrians affect directly the turning left or right flows at signalized intersections, the turn flow is an important factor. At the studied intersection, the proportion of the turn flows (turning right and turning left) is high, up to $79.4 \%$ and $80.1 \%$ for approach 1 (traffic from Oteo bridge) and approach 2 (traffic from Nagaoka station), respectively. The proportion of turn movement in comparison with that of through movement at the studied intersection is as illustrated in Figure 5. 


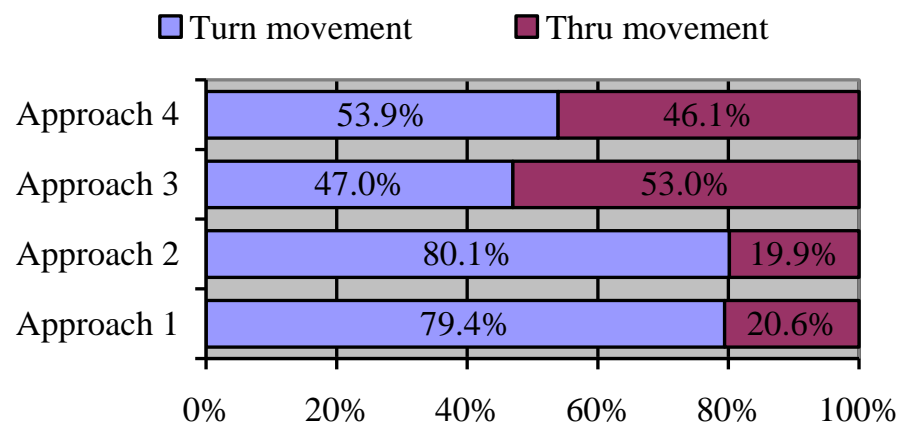

Figure 5. Turn Proportion at the Studied Intersection

Four entry approaches of the studied intersection have zebra crossings for pedestrians. The research defines average hourly pedestrian trip as the average number of trips making by pedestrians to cross at the four crossings in one hour. A pedestrian trip is counted only if a pedestrian finishes a crossing at a zebra crossing. A video based software (SEV) [13] is used to collect walking speed, pedestrian flow, etc. The dimensions of pedestrian crossings at four intersections are as illustrated in Figure 6 and Table 4.

Table 4. The Dimension of Pedestrian Crossings

\begin{tabular}{|c|c|c|c|}
\hline Dimensions & A $(\mathrm{m})$ & B $(\mathrm{m})$ & $\mathrm{C}(\mathrm{m})$ \\
\hline Direction 1-2 & 14.3 & 4.5 & 2.0 \\
\hline Direction 3-4 & 17.3 & 4.5 & 2.0 \\
\hline
\end{tabular}

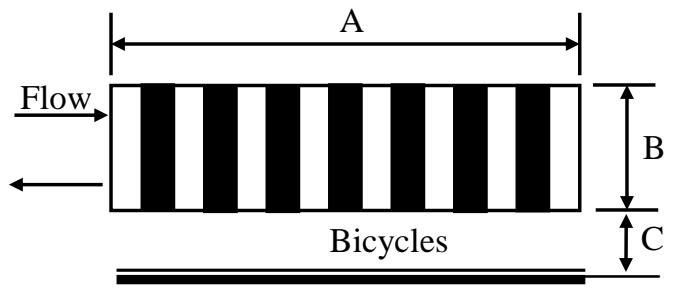

Figure 6. The Dimensions of Pedestrian Crossings

At the studied intersection, effects on traffic flow are caused not only pedestrians but also cyclists. According to the observation, the shares of pedestrians and cyclists are $57 \%$ and $43 \%$, respectively. The pedestrian speeds and bicycle speeds are determined based on the recorded videos and field observation. The distributions of observed speeds are satisfied with Kolmogorov-Smirnov Test. The data analysis shows that, the average speed of bicycles is 3.4 $\mathrm{m} / \mathrm{s}$ with the deviation of 0.9 . For the sake of simplicity, the research simulates crossing cyclists as crossing pedestrians with different sizes and walking speeds in PARAMICS. With Kolmogorov-Smirnov Test (test statistic $\mathrm{Z}=0.968$; Most Extreme difference $\mathrm{D}=0.059$; and $\mathrm{P}$ value $=0.306>0.05$ ), the pedestrian speed can be considered a normal distribution as shown in Figure 7. 


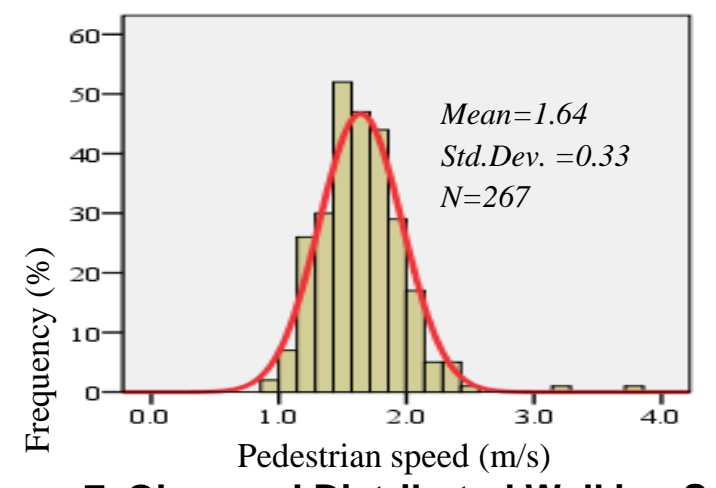

Figure 7. Observed Distributed Walking Speed

\section{Result and Analysis}

\subsection{Simulation of the Base Scenario}

To evaluate the model performance, the paper uses relative error (RE) as a criterion. The definition is expressed as follows:

$$
R E=\frac{\left|V_{\text {sim }}-V_{o b s}\right|}{V_{\text {obs }}}
$$

where,

$$
\begin{aligned}
& \text { VSim : Simulation values } \\
& \text { VObs : Observation values }
\end{aligned}
$$

The flow rates were compared between the simulation values and observation ones for every 5 min interval. The simulation values and corresponding observation ones are generally approximate. The relative error is rather small as shown in Figure 8.

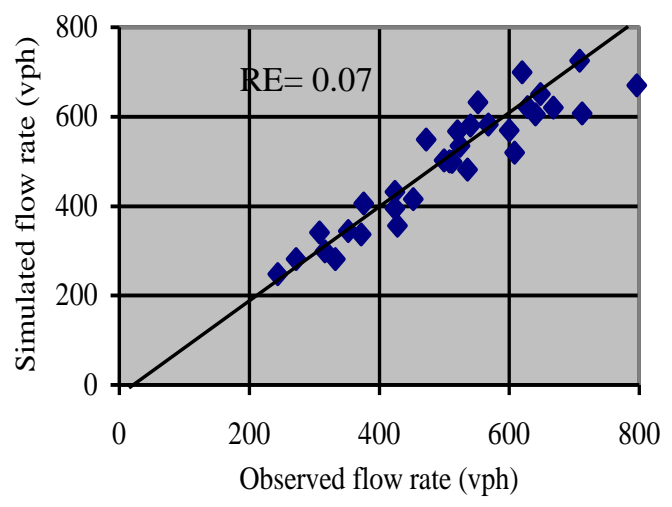

Figure 8. Traffic Flow Validation

As for pedestrian flow, the research compared the pedestrian flows at each signal cycle of the studied intersection. The data of simulated pedestrian flow is directly counted from the simulation animation with different random seeds. The values of simulated pedestrian flows and observed pedestrian flows (person per minute ( $\mathrm{ppm})$ ) distributes closely along the 45degree line with low relative errors as in Figure 9. 


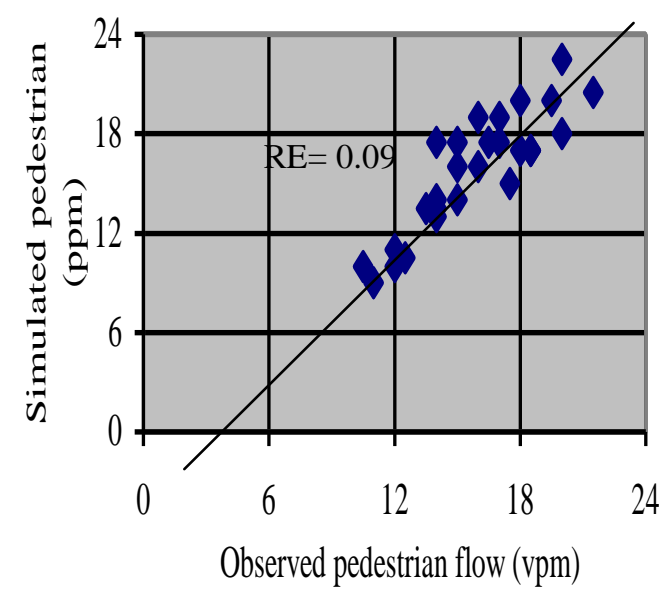

Figure 9. Pedestrian Flow Validation

\subsection{Pedestrian based LOS Analysis}

In this subsection, the research firstly compares the intersection level of service (LOS) at the studied intersection under various crossing pedestrian demands. Keeping the current observed traffic flow, the research changes the pedestrian demand and analyses intersection LOS based on the vehicle delay at the intersection. The results show that the intersection LOS in the base scenario with normal crossings varies significantly with respect to the hourly pedestrian trip. However, the figure changes insignificantly in the scenario of scramble crossings. The details are as follows:

4.2.1. The Base Scenario with Normal Pedestrian Crossings: Because of the direct impedance of crossing pedestrians at the signalized intersection, the delay imposing on turning vehicles causes changes in LOS values of the intersection. As shown in Figure 10, the intersection signal initially determines the intersection LOS at C level when there is no impedance from crossing pedestrian. When the hourly pedestrian trip increases, the intersection LOS switches from C to D, E or F depending on the value of hourly pedestrian trip. If the hourly pedestrian trip at the intersection is higher than 4000 (persons per hour (pph)) and lower than 6000 (pph), the intersection LOS is at level $\mathrm{D}$. The curve of vehicle delay increases rapidly when the hourly pedestrian trip is larger than 5000 (pph). The effects of crossing pedestrians can turn the intersection LOS to the level $\mathrm{F}$ with high values of hourly pedestrian trip as shown in Figure 10. The variance of the result is represented by the standard deviation in this research. With low values of pedestrian trip, the standard deviations are small, around 2.2 (s). This value increases when the pedestrian demand increases, up to $8.4(\mathrm{~s})$. 


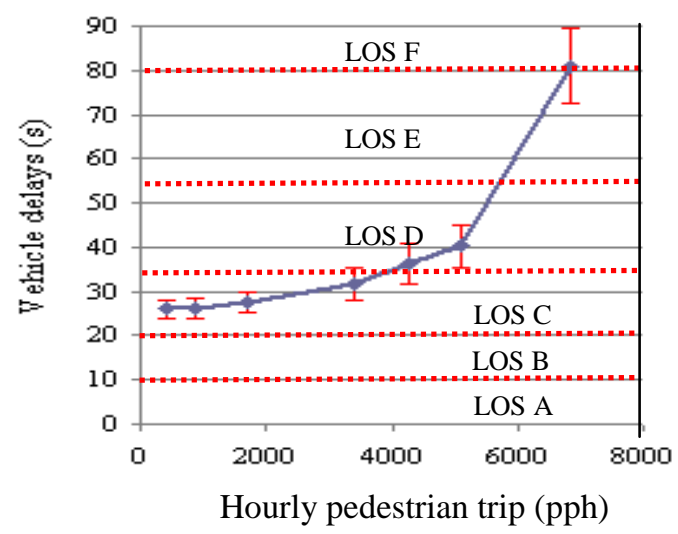

Figure 10. Pedestrian based LOS for the Normal Crossings

4.2.2. The Scramble Scenario: The scramble crossings with private signal phases for pedestrian helps pedestrians traverse the intersection safely. Even when the hourly pedestrian trip increases, the vehicle delay increases slowly. The standard deviation changes slightly. This deviation is directly proportional to the pedestrian demand. The maximum value of deviation is 1.96 (s) in this study. As shown in Figure 11, the LOS of the intersection almost does not change. The intersection LOS is at D level regardless of the magnitude of the hourly pedestrian trip at the intersection. The details are as shown following.

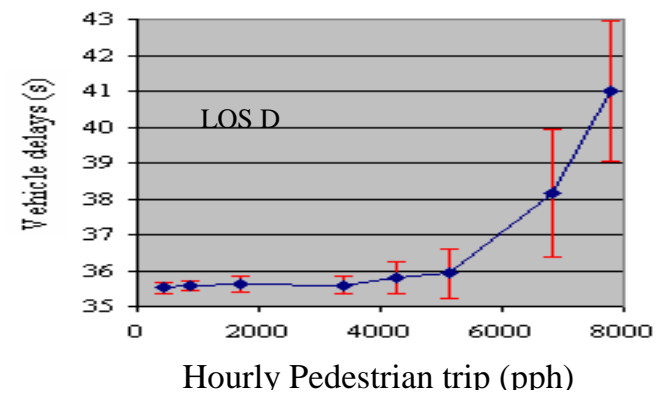

Figure 11. Pedestrian based LOS for Scramble Crossings

\subsection{Suitable Areas for Scramble Crossings}

Although the normal pedestrian crossings can initially hold the intersection LOS at C level, much better than that in the scenario of scramble crossings with D level when hourly the pedestrian trip is low, the scramble crossings give pedestrian a safe facility of walking. In addition, the scramble crossings have a stable LOS even when the number of hourly pedestrian trip increases. Except the aspect of safety, the vehicle delay is an important factor to decide which crossing is suitable for a signalized intersection. With the current traffic situation, the decision on choosing normal pedestrian crossings or scramble crossings in terms of vehicle delay at the intersection is investigated in this part. As shown in Figure 12, the critical point at which the vehicle delays in the scenarios of normal pedestrian crossings and scramble crossings are the same divides the graph into two parts. The left part $(\mathrm{P}<4800(\mathrm{pph}))$ where the vehicle delay in the normal crossing scenario is less than that in the scenario of scramble crossing is suitable for normal pedestrian crossing deployment. On the contrary, the 
right part in which the hourly pedestrian trip is higher than 4800 is proper for the deployment of scramble crossings. In this area, the scramble crossings reduce the vehicle delay at the intersection significantly compared with the normal pedestrian crossings. The detail is as shown in Figure 12.

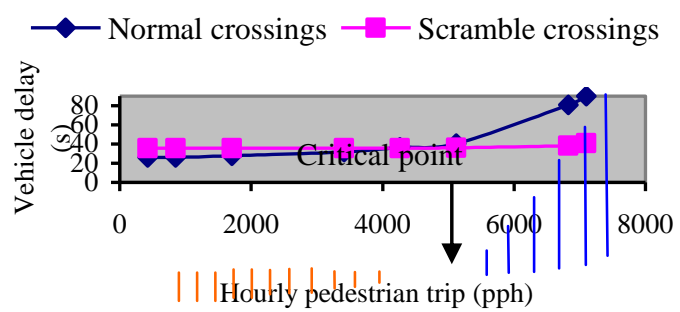

Figure 12. Proper Areas for Scramble Crossings

\subsection{Traffic Flow based LOS Analysis}

Not only pedestrians, but also traffic flow affects the LOS of the intersection. To understand the effects of pedestrian more comprehensively in comparison with that of traffic flow, the research conducts an analysis of intersection LOS based on the variation of traffic flow. When a green light starts, thru traffic flow can travel smoothly through the intersection. However, the turn flow is impeded by crossing pedestrians. The research investigates the effects of traffic flow by increasing the turn flow and keeping the thru traffic flow unchanged. The average traffic flow is the average of traffic flow on each approach of the intersection. At the surveyed situation, the average traffic flow and hourly pedestrian trip are 501 (vph) and 853 (pph), respectively in this study. This pedestrian demand is the observed demand during the peak hour at the studied intersection. As shown in Figure 13, even the traffic flow increases, vehicle delay in the scenario of scramble crossing is higher than that in the normal crossing scenario. The normal pedestrian crossing is more suitable than scramble crossing in these scenarios. In other words, scramble crossings are not proper if considering the traffic flow only. When investigating into which pedestrian crossings, normal crossings or scramble crossings, is suitable for an intersection, it is better to consider the pedestrian demand instead of traffic flow at the intersection. The details of each scenario are as shown in Figure 13.

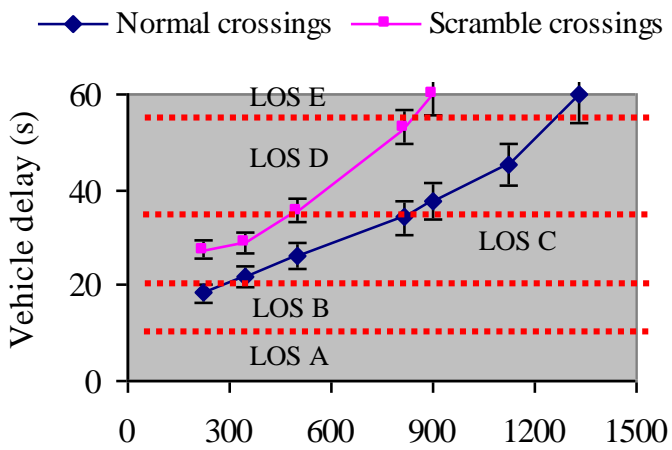

Average traffic flow (vph)

Figure 13. Traffic Flow based LOS 


\subsection{Sensitivity Analysis}

At the studied intersection, pedestrian demands as well as traffic flow varies hour to hour or day by day. An investigation into the effects of pedestrian and traffic flow on the decision of choosing the scramble crossings is necessary. Called the improvement of scramble crossings compared with the normal crossings in terms of vehicle delay as I, the definition is expressed as follows:

$$
\begin{aligned}
& P=\frac{D_{0}-D_{s}}{D_{0}} \cdot 100 \% \\
& I(\%)=\left\{\begin{array}{lll}
P & , \text { If } \mathrm{P} \geq 0 \\
0 & , \text { Otherwise } & \text { (Scramble is BENEFICIAL) }
\end{array}\right.
\end{aligned}
$$

where,

P: Percentage of delay difference $(\%)$

D0: The vehicle delay in the normal scenario (s)

DS: The vehicle delay in the scramble scenario (s)

I: The improvement of the scramble crossing in comparison with the normal pedestrian crossings $(\%)$

As shown in Figure 14, the scramble crossing can improve the intersection delay by up to $35.74 \%$ (with the standard deviation of $6.4 \%$ ) in scenarios of high pedestrian demand. The figure confirms the stronger effect of pedestrians on the performance of the intersection in comparison with that of the traffic flow. Indeed, with high values of hourly pedestrian trip (around 6000pph), the scramble crossing is proper for nearly all of the traffic flow conditions. Moreover, Figure 14 shows that, in scenarios of high traffic flow (greater than 1200vph), the scramble crossing is suitable for the minimum hourly pedestrian trip of 2000 pedestrians per hour. The lower the average traffic flow is, the higher the pedestrian demand is required for choosing the scramble crossing in comparison with the normal crossings. This is proper to previous studies on pedestrian that as a prerequisite for installing a scramble at an intersection, Transportation Services looks for a volume of at least two thousand pedestrians per hour, sustained over an eight-hour period [19]. The details of the analysis are as shown in Figure 14.

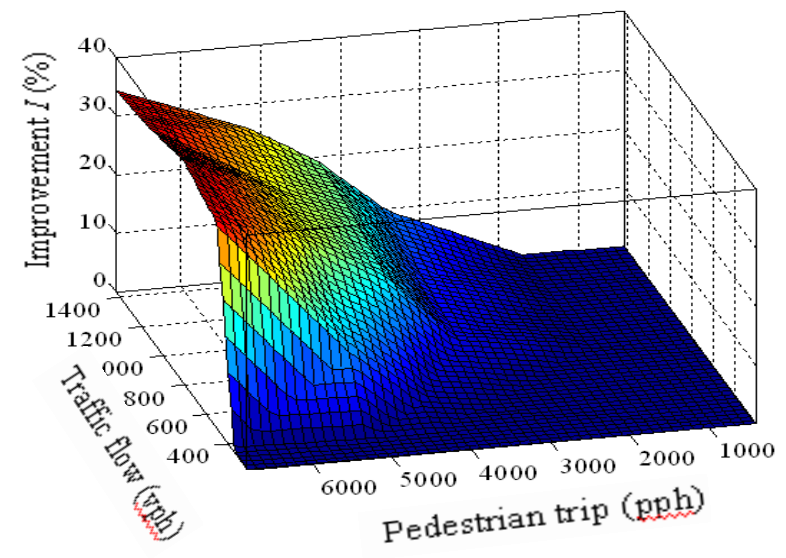

Figure 14. Sensitivity Analysis for Choosing Scramble Crossings 
However, the blue area in which the pedestrian demand is low (less than 2000 (pph) for example) as shown in Figure 14 is not suitable for scramble crossings in terms of delay reduction. Unlike the cases in which the scramble is beneficial (green, yellow and red areas in Figure 14), an increase in traffic flow makes the scramble worse than the normal crossing (Figure 13). It is suggested to introduce normal crossings instead of scramble in this area.

\section{Conclusion}

The research aims at two targets. The first target is to design and simulate the scenario of scramble crossings based on current normal pedestrian crossings at a signalized intersection in Nagaoka city of Japan. The second target is the analysis of effects of the scramble crossings and that of the normal one under various situations of traffic flow as well as pedestrian demand. The intersection LOS shows that the scramble crossings are more stable than the normal pedestrian crossing in terms of intersection performance when the pedestrian demand changes. The analysis confirms the important role of the pedestrian demand in the decisions of choosing the scramble crossings. The higher the pedestrian demand is, the more advantageous the scramble crossings are. The scramble crossings can reduce the vehicle delay significantly, by up to $35.74 \%$ compared with the normal pedestrian crossings.

The research has several shortcomings needed to be improved. The research evaluates the scramble crossings in terms of vehicle delay reduction only. The aspect of pedestrian delay and safety is an important factor that should be concerned in next research studies. Intuitively, the number of conflictions between vehicles and pedestrians in the scenarios of normal crossings and scramble crossings are quite different. Because of the exclusive signal for pedestrian in the scenario of scramble crossings, the number of conflictions in this scenario is lower than that in the normal crossing scenario if considering under the same situations of traffic and pedestrian demand. Therefore, the safety level or the reduction of pedestrian delay in the scenario of scramble crossings is better than in the normal crossings. This kind of safety should be studied in future research. In addition, the results of simulation-based analysis require real data of scramble crossing deployments to have a more realistic evaluation. Moreover, the fixed signal in this study should be complicated to become actuated or adaptive $([10,11,17])$ to clarify the benefit of scramble crossing. The points should be investigated more in further studies to understand comprehensively about the benefit of scramble crossings in developing a pedestrian-friendly environment as well as encouraging walking in urban areas.

\section{Acknowledgements}

The authors highly appreciated all members of Transportation Lab for data collection as well as valuable suggestions from anonymous authors in improving the quality of the paper.

\section{References}

[1] A. K. Bechtel, K. E. MacLeod and D. R. Ragland, "Oakwood Chinatown Pedestrian Scramble: An Evaluation”, UC Berkeley Traffic Safety Centre, (2003).

[2] C. Pretto, H. Cybis and A. Jacobsen, "A multi-layer simulation model for vehicle and pedestrian interaction", Compendium of Papers CD-ROM, the 89th Annual Meeting of the Transportation Research Board, Washington D.C., (2011) January 22-26.

[3] X. Chen, C. Shao and H. Yue, "Influence of Bicycle Traffic on Capacity of Typical Signalized Intersection", Tsinghua Science and Technology, ISSN 1007-0214 10/14, vol. 12, no. 2, (2007), pp. 198-203. 
[4] D. Cheng, Z. Tian and H. Liu, "Implementing actuated signal controlled intersection capacity analysis with pedestrians", Compendium of Papers CD-ROM, the 87th Annual Meeting of the Transportation Research Board, Washington D.C., (2008) January 13-17.

[5] P. Eleonora, Y. George and G. John, "A critical assessment of pedestrian behaviour models", Transportation Research Part F 12, (2009), pp. 242-255.

[6] M. M. Ishaque and R. Bm Noland, "Micro-simulation Comparisons of Alternative Signalized Pedestrian Crossings", Paper submitted for presentation at the Transportation Research Board Annual Meeting, Washington D.C., (2006) January 22-26.

[7] J. P. Pitaksringkarn, "Implementation of the first pedestrian scramble in downtown San Diego", California, Proceedings of the Eastern Asia Society for Transportation Studies, vol. 5, (2005), pp. 2504-2515.

[8] K. Hagita, T. Hagiwara and H. Hamaoka, "Analysis of Driver's Visual Conditions in Right-Turning Accidents involving Pedestrians at Signalized Intersection in Japan", Compendium of Papers CDROM, the 89th Annual Meeting of the Transportation Research Board, Washington D.C., (2011), January 22-26.

[9] K. L. Kotkar, R. Rajat and C. Satish, "Pedestrian Flow Characteristics in Mixed Traffic Conditions", Journal of urban planning and development (ASCE), vol. 136, no. 1, (2010), pp. 23-33.

[10] W. Lin, H. K. Lo and L. Xiao, "A Quasi-Dynamic Robust Control Scheme for Signalized Intersections", Journal of Intelligent Transportation Systems, vol. 15, no. 4, (2011), pp. 223-233.

[11] H. K. Lo and A. H. F. Chow, "Control Strategies for Oversaturated Traffic", Journal of Transportation Engineering, vol. 130, no. 4, (2004), pp. 466-478.

[12] R. V. Mark, "Scramble and Crosswalk Signal Timing, Transportation Research Record 1636", accessed on Mar. 18th 2013, available at http://www.enhancements.org/download/trb/1636-013.PDF.

[13] C. C. Minh, "Analysis of motorcycle behaviour at Midblocks and Signalized intersections", Ph.D thesis, Nagaoka University of Technology, (2007).

[14] S. C. Mohsin and R. Prakash, "Capacity Analysis of Signalised Intersection using Microsimulation", 32nd Australian Transport Research Forum, (2009).

[15] M. I. Muhammad and B. N. Robert, "Trade-offs between vehicular and pedestrian traffic using micro-simulation methods", Transport Policy, vol. 14, (2007), pp. 124-138.

[16] M. R. Nagui and S. E. Brian, "Pedestrian Impedance of Turning-Movement Saturation Flow Rates Comparison of Simulation", Analytical, and Field Observations, Transportation research Record 1578, Paper No. 971239, (1997), pp. 56-63.

[17] G. F. Newell, "Theory of Highway Traffic Signals", University of California, Berkeley, CA.: Institute of Transportation Studies, Course Notes, (1989).

[18] Quadstone PARAMICS, PARAMICS User Guide - Version 6.9.1, Quadstone Limited, Edinburgh, UK, (2013).

[19] B. Rajnath and T. Craig, "Pedestrian Scramble Crossings - A Tale of Two Cities", accessed on Mar. 18th 2013, available at http://www.toronto.ca/transportation/walking/pdf/pedestrian_scramble _crossings.pdf

[20] T. R. Richard and K. S. Shashi, "Integrated Systems Methodology for Pedestrian Traffic Flow Analysis", Transportation research Record 1578, Paper No. 971195, pp. 30-37.

[21] S. Clark., "Scramble Pedestrian Crossings", Department for Transport, Energy and infrastructure, Government of South Australia, (1998).

[22] K. Sano, Y. Suga, R. Pairoj and W. Wisinee, "A study on the benefit caused by the improvement of the punctuality of the local bus service", Journal of the Eastern Asia Society for Transportation Studies, vol.7, (2007), pp. 1575-1583.

[23] S. Acharjee, L. Kattan and R. Tay, "A Pilot Study on Pedestrian Scramble Operations in Calgary", Paper submitted for presentation at the Transportation Research Board Annual Meeting, Washington D.C., (2009) January 10-15.

[24] T. Hagiwara, H. Hamaoka, K. Hagita, Y. Tabata, D. Uchibori and H. Takagi, "Analysis of Traffic Conflicts between Right-Turning Vehicles and Pedestrians/Cyclists at an Urban Signalized Intersection", Compendium of Papers CD-ROM, the 89th Annual Meeting of the Transportation Research Board, Washington D.C., (2010) January 10-14.

[25] N. Takashi, "Four species CA model for facing pedestrian traffic at rush hour", Applied Mathematical Modelling, vol. 36, (2012), pp. 702-711.

[26] TRB, Highway Capacity Manual, National Research Council, Washington, D.C.,USA, (2010).

[27] W. Brilon and T. Miltner, "Capacity and Delays at Intersections Without Traffic Signals", Compendium of Papers CD-ROM, the 84h Annual Meeting of the Transportation Research Board, Washington D.C., (2005) January 09-13. 
[28] X. Chen, C. Shao and H. Yue, "Influence of Pedestrian traffic on capacity of right-turn movements at signalized intersections", Compendium of Papers CD-ROM, the 87th Annual Meeting of the Transportation Research Board, Washington D.C., (2008) January 13-17.

[29] Z. Zhou, G. Ren, W. Wang, Y. Zhang and W. Wang, "Pedestrian Crossing Behaviors at Signalized Intersections: Observational Study and Survey in China", Compendium of Papers CD-ROM, the 89th Annual Meeting of the Transportation Research Board, Washington D.C., (2011) January 22-26.

[30] J. Yang, W. Deng, J. Wang, Q. Li and Z. Wang, "Modeling pedestrians_ road crossing behavior in traffic system micro-simulation in China", Transportation Research Part A 40, (2006), pp. 280-290.

[31] T. Kardi, "Application of microscopic pedestrian simulation model", Transportation Research Part F, vol. 9, (2006), pp. 15-27.

[32] V. P. Sisiopiku and D. Akin, "Pedestrian behaviors at and perceptions towards various pedestrian facilities: an examination based on observation and survey data, Transportation Research Part F, vol. 6, (2003), pp. 249-274.

[33] Z. Yang and R. F. Benekohal, "Pedestrian crossing phase optimization based on minimizing vehicle and pedestrian delays by genetic algorithm", Compendium of Papers CD-ROM, the 90th Annual Meeting of the Transportation Research Board, Washington D.C., (2011) January 22-26.

\section{Authors}

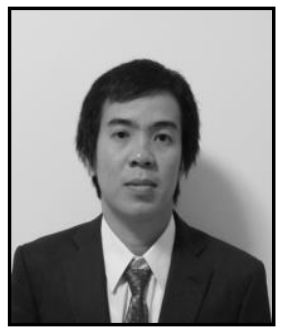

Dr. Tran Vu TU, he is a postdoctoral researcher at Niigata University. He achieved a doctoral degree in Transportation Engineering at Nagaoka University of Technology in Japan. Before coming to Japan, he worked as a lecturer in the department of civil engineering, Saigon Technology University (STU), Vietnam. His research interests are traffic engineering, traffic simulation, Transit Priority System, network analysis, discrete choice model, mathematical optimization. He has published numerous journals and proceedings as well as co-authored many research papers in the related fields.

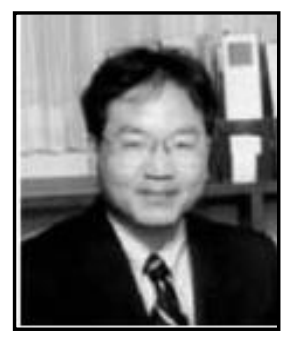

Dr. Kazushi SANO, he is an Associate Professor in the Department of Civil and Environmental Engineering at Nagaoka University of Technology (NUT) in Japan. Dr. Sano received a doctoral degree in Doctor of Engineering in Traffic Engineering and Land Planning. He has published numerous papers and reports in such journals and proceedings as Academic Journal, Advanced Transgraft Telematics/Intelligent Vehicle-Highway Systems, or Application of Artificial Intelligence in Engineering. His research interests are in the area of traffic engineering, traffic simulation, public transport system, and land planning. 